\title{
A NOTE ON MODULES AND RADICALS
}

\section{IRVIN ROY HENTZEL ${ }^{1}$}

Introduction. Andrunakijevic and Rjabuhin have shown in [1] that every Kurosh-Amitsur radical for associative rings can be described in the language of modules in much the same way as the Jacobson radical.

An examination of Andrunakijevic and Rjabuhin's arguments shows that all but one of their results can be obtained by omitting one of their conditions. Since that condition is the most difficult one to verify in practice, this raises the question of whether one can obtain all the results in [1] without it. In this note we show that this will not be possible in general.

We also give a new and short proof of the main result in [1].

1. Preliminary definitions and constructions. All rings are assumed to be associative and when we say that $M$ is an $A$-module, it is assumed $M A \neq 0$.

If $M$ is an $A$-module then $(0: M)_{A}=\{a \in A \mid M a=0\}$ is an ideal of $A$. If $\Sigma_{A}$ is a collection of $A$-modules then an ideal, kern $\Sigma_{A}$, of $A$ is defined by: kern $\Sigma_{A}=\bigcap\left\{(0: M)_{A} \mid M \in \Sigma_{A}\right\}$ if $\Sigma_{A} \neq \varnothing ; \operatorname{kern} \Sigma_{A}=A$ if $\Sigma_{A}=\varnothing$. We say that $\Sigma_{A}$ is faithful if kern $\Sigma_{A}=0$.

If $\beta$ is a homomorphism of a ring $A$ onto a ring $A^{\beta}$, then (1) each $A^{\beta}$-module $M$ can be considered as an $A$-module, (2) each $A$-module such that kern $\beta \subseteq(0: M)_{A}$ becomes an $A^{\beta}$-module. We note that $(0: M)_{A}=\beta^{-1}\left[(0: M)_{A}^{\beta}\right]$.

We recall from radical theory, the upper radical $U_{\Re}$ constructed from a regular class $\mathfrak{T}$, see [2] or [3].

2. The conditions of Andrunakijevic and Rjabuhin. Suppose that to each ring $A$ a class $\Sigma_{A}$ of modules has been assigned and let $\Sigma$ be the union of the $\Sigma_{A}$, taken over all rings $A$. We consider four conditions which $\Sigma$ may satisfy.

P.1. For each ideal $B$ of $A, M \in \Sigma_{A / B} \Rightarrow \mathbb{M} \in \Sigma_{A}$.

P.2. $M \in \Sigma_{\boldsymbol{A}}$ and $B$ an ideal of $A$ such that $(0: M)_{A} \supseteq B \Rightarrow \mathbb{M} \in \Sigma_{\boldsymbol{A} / \boldsymbol{B}}$.

P.3. $\Sigma_{A}$ faithful $\Rightarrow \Sigma_{B} \neq \varnothing$ for each ideal $B \neq 0$ of $A$.

P.4. $\Sigma_{B} \neq \varnothing$ for each ideal $B \neq 0$ of $A \Rightarrow \Sigma_{A}$ is faithful.

We shall say that $A$ is a $\sigma$-ring if $\Sigma_{A}=\varnothing$, and we let $\mathcal{L}(\Sigma)$ be the class of rings $A$ for which $\Sigma_{A}$ contains a faithful module $M$, that is, a

Received by the editors July 21, 1967.

1 This paper was written while the author held an NSF Graduate Fellowship. 
module $M$ such that $(0: M)_{A}=0$. Of course, for each $A$ in $\mathscr{L}(\Sigma)$, we have kern $\Sigma_{A}=0$.

THEOREM. If $\Sigma$ satisfies $\mathrm{P} .1, \mathrm{P} .2$, and $\mathrm{P} .3$, then $\sigma$ is a radical property and in fact is the upper radical determined by $\mathcal{L}(\Sigma)$. Moreover, for each ring $A, \sigma(A) \subseteq$ kern $\Sigma_{A}$, where $\sigma(A)$ denotes the $\sigma$-radical of $A$.

Proof. It is easy to show that $\sigma$ is a radical property by showing that $A$ is a $\sigma$-ring if and only if $A$ is a $\bigcup_{\mathscr{L}(\Sigma)}$ ring for the regular class $\mathfrak{L}(\Sigma)$. If $I$ is a $\sigma$-ideal of $A$ and $I \nsubseteq \not$ kern $\Sigma_{A}$ then for some $M \in \Sigma_{A}$ $\left[(0: M)_{A}+I\right] /(0: M)_{A}$ is a nonzero ideal of $A /(0: M)_{A}$. Since $A /(0: M)_{A} \in \mathscr{L}(\Sigma)$ and $\mathscr{L}(\Sigma)$ is a regular class $\left[(0: M)_{A}+I\right] /(0: M)_{A}$ has a homomorphic image in $\mathscr{L}(\Sigma)$. Thus $I$ has a homomorphic image in $\mathscr{L}(\Sigma)$ which contradicts the fact that $I$ is $U_{\mathscr{L}(\Sigma) \text {-radical. }}$

REMARK. Andrunakijevic and Rjabuhin proved that if $\Sigma$ satisfies P.4 as well then $\sigma(A)=\operatorname{kern} \Sigma_{A}$. In the next section we give an example of a class $\Sigma$ which satisfies P.1, P.2, and P.3, but for which $\sigma(A) \neq \operatorname{kern} \Sigma_{A}$.

3. A counterexample. Let $I$ be a regular class of nonzero rings. For each $T \in J$ let $M_{T}$ be a fixed, but arbitrary, faithful $T$-module. If $A$ is an arbitrary ring we put $\Sigma_{A}=\left\{M_{T} \mid T \in J\right.$ and $T$ is a homomorphic image of $A\}$. Then $\Sigma_{A}$ satisfies P.1, P.2, and P.3.

For the counterexample, let $Z_{p}$ be the trivial ring on the cyclic group of order $p, p$ a prime. Then the class $J$ consisting of $Z_{p}$ alone is a regular class. Construct $\Sigma$ as above. Let $A$ be the trivial ring on the cyclic group of order $p^{2}$. Then $\sigma(A)=0$, but kern $\Sigma_{A}=Z_{p}$.

The author wishes to thank Professor T. Anderson for his helpful advice and criticism of the manuscript.

\section{BiBLIOGRAPHY}

1. V. A. Andrunakijevic and Ju. M. Rjabuhin, Modules and radicals, Dokl. Akad. Nauk SSSR 156 (1964), 991-994 = Soviet Math. Dokl. 5 (1964), 728-731,

2. N. J. Divinsky, Rings and radicals, Math. Expositions no. 14, Univ. of Toronto Press, 1965.

3. A. G. Kurosh, Radicals of rings and algebras, Mat. Sb. N.S. 33 (75) (1953), 13-26.

The University of Iowa 